\title{
Intervención en el Castillo de Biar. Consolidación de una ruina como alternativa posibilista en la defensa del patrimonio
}

Biar's Castle intervention. Consolidation of a ruin as a possibilist alternative in the defense of heritage

\author{
Miguel del Rey ${ }^{a}$, Antonio Gallud ${ }^{\text {b }}$ \\ Instituto Universitario de Restauración del Patrimonio - Universitat Politècnica de València, Valencia, Spain \\ a juanmigueldelrey@gmail.com; ${ }^{\mathrm{b}}$ agallud@upv.es
}

\begin{abstract}
Consolidation of ruin and didactic recovery of the castle's profile dominating the landscape. Almohad fortress that should have been recorded at the beginning of the powerful existing tower, surrounded by a protective wall with adarve, all on steep rocks.

The fortress is transformed over time, being in service as a defense between Muslim Spain and Christian Spain in the twelfth and thirteenth centuries, to later, be a point of friction between the Kingdoms of Castilla and Aragón, remaining active until the mid-sixteenth century, with an intervention in the nineteenth century during the Carlist wars. Is located on the top of a hill at $745 \mathrm{~m}$ altitude, next to the town. The orography marks a deep slope to the northeast, more than $100 \mathrm{~m}$ high, while to the west, falling towards the population, the slope is smoother. Because of its situation, the castle has a very important visual impact, so landscape considerations acquire a special meaning.

The intervention is partial on the second walled enclosure and in total ruin, proposing an eloquent restoration that allows to approach its complex history and the construction techniques used, within a strong economy of means in the project and subsequent maintenance. We can restore the image of the courtyard, its spatiality and know the remains of existing buildings. Both, the remnants emptying of crashes, and the restoration of the traces of the internal walls, the various heights of the walls and their guard steps, allow us to understand the whole along the time. The undoubted visual and landscape interest of Biar Castle is a relevant aspect of the intervention.
\end{abstract}

Keywords: Castle, fortification, Biar, landscape value.

\section{Introducción}

El proyecto de consolidación del Castillo de Biar se redacta por el Grupo de Investigación PAISAR "Paisaje y Arquitectura Rural" del Instituto de Restauración de la Universidad Politécnica de Valencia a instancias del Ministerio de Cultura. Siendo los responsables del mismo los profesores D. Miguel del Rey, D. Antonio Gallud, y D. Ignacio Fuster.
Pretende consolidar y hacer una lectura ajustada del primer recinto de la muralla del Castillo con dos intenciones complementarias: Consolidar la ruina de las fábricas -con el peligro que supone para visitantes y habitantes de las partes altas de la población-, a la vez que poner en valor y trasmitir el interés de estas arquitecturas y estas formas, en sus diferentes momentos, al público interesado, potenciando una forma coherente y 
evocadora de lo que fue la forma, dos dimensiones y el perfil del castillo en el paisaje.

Para la primera de las acciones a desarrollar se disponía de un presupuesto muy ajustado, por lo cual hubo que actuar con una fuerte economía de medios que incidió en el proyecto, abandonando cualquier pretensión de revivir formas represtinadas en caso alguno; más bien nos interesó una consolidación elocuente de la muralla capaz por ella misma de trasmitir la emoción de unas formas y unas arquitecturas pretéritas.

Se han conseguido conocer, identificar, analizar la naturaleza de sus fábricas y restaurar los elementos primigenios que configuraron la cerca islámica del siglo XII, los diversos niveles de ampliación de los muros hasta llegar a la máxima altura en las obras de los siglos XV o XVI; además de hacer evidente la planta original, la inexistencia primigenia de torres esquineras y la sucesiva incorporación de las mismas en las ampliaciones y transformaciones del castillo, ya en época cristiana. Se ha logrado poner en valor todos estos elementos y etapas históricas dentro de un proyecto didáctico y lógico, con una restauración que se entiende disciplinada.

\section{El Castillo y su paisaje}

Biar se sitúa en la falda de una colina coronada con su Castillo. Desde la distancia encontramos un paisaje de campos de olivos sobre los que se eleva la colina que en su ladera sur sitúa el pueblo. Sobre él, separado del caserío se encuentra el castillo, con doble cerco de murallas, y sobre ellos se eleva la torre celoquia almohade del siglo XII, germen del castillo.

Al norte vemos la roca desnuda y un gran corte vertical se funde con la muralla del castillo y su torre. Desde el resto de orientaciones se ve con claridad el castillo con sus muros en posición dominante. A media altura un bosque de pinos separan los cercos.

\section{Arquitectura del Castillo}

El castillo tiene doble recinto amurallado construido en fechas muy distintas, formados ambos por lienzos limitados por torres semicirculares, coronando el conjunto la torre celoquia, empla- zada en el punto más elevado del recinto y formando parte de su perímetro defensivo norte.

La entrada al castillo se produce por la zona más próxima a la población, con puerta de sillería de arco de medio punto en el exterior y carpanel interior, flanqueada por sendas torres. El lienzo donde se ubica la puerta abarca dos frentes del castillo, por ser la zona más vulnerable, ya que los otros lados se sitúan sobre el brusco desnivel descrito anteriormente. Entre este antemuro y la muralla interior se emplaza uno de los aljibes de la fortificación.

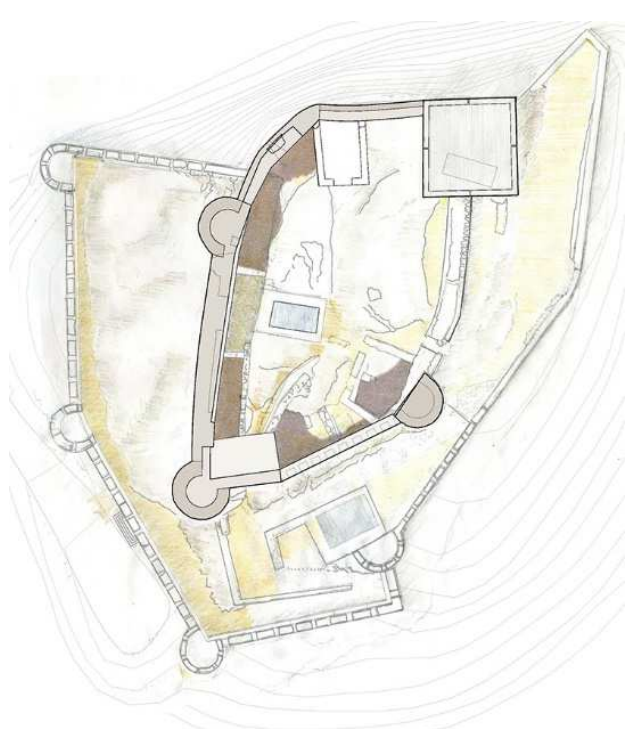

Fig. 1. Planta del Castillo (Miguel del Rey, Antonio Gallud, 2010).

La torre celoquia tiene tres niveles con puerta de arco de medio punto en el exterior y rebajado en el interior. Su basamento es de mampostería sobre la que se construye el tapial, de cajas de cuatro palmos por un espesor medio de $135 \mathrm{~cm}$. de espesor, con altura total de $19 \mathrm{~m}$. Muestra en su interior elementos de gran interés, espacios abovedados en cada nivel. Su remate está coronado por matacanes en el centro de cada uno de sus lados.

El castillo presenta un estado muy distinto en cada una de sus partes. La torre celoquia fue restaurada en su momento, siendo un elemento muy relevante en el conjunto. El recinto externo, con 
fábricas deterioradas en parte, se encontraba en mejor estado que el interno. Los muros del recinto interno presentaban un importante estado de ruina e incluso habiendo desaparecido alguno de los tramos. El tramo que cierra a poniente es el único que mantenía una cierta prestancia y nos mostraba la altura del adarve de la última de las épocas en que estuvo en servicio el castillo.

El recinto interior, sobre el que se actúa, cuenta con una serie de paños de muro que quiebran en tres torres, hasta cerrar abrazando a la torre celoquia. La forma de estas torres perimetrales es semicircular en las laterales y casi cilíndrica en la situada junto a la puerta de acceso a este recinto. En la última época carecía de almenado, con restos de parapeto en algunos puntos. El ingreso al recinto superior es a través de un arco de sillería de medio punto, que da paso a una sala rectangular cubierta con bóveda que provoca un recodo para acceder al patio interno del castillo.

Los lienzos del primer recinto están construidos con mampostería y, sobre este, se dispone un estrecho adarve con merlones y troneras. Los muros del recinto interno, objeto de este proyecto, son de etiología muy diversa, con fragmentos de tapiales y láminas de mampostería, con muy distintas secciones y acabados diversos que incluyen adarves de distintos anchos, restos de antiguos merlones y fragmentos de parapetos en algunos casos.

\section{Objeto de la intervención}

\subsection{Protección de los restos arquitectónicos}

El primer objetivo del proyecto es la conservación y consolidación de los restos arquitectónicos, tanto correspondientes a los muros de perímetro, como a las fábricas interiores existentes. Dado el grado de erosión y deterioro que presentaban la mayor parte de los muros, se propuso inicialmente la limpieza y consolidación de sus paramentos. Se estudió cada fragmento de forma particularizada para definir la estrategia de intervención adecuada a cada caso.

\subsection{Reconfiguración del espacio del recinto interior}

La consolidación de los restos arquitectónicos permite restablecer la espacialidad del patio de armas permitiendo comprender la idea de recinto cerrado de esta parte del castillo, frente a la actual configuración informe y abierta que presentaba en esta última época. También hay que señalar la importancia que adquiere la presencia y definición del paso de ronda perimetral, lo que contribuye a la comprensión funcional de cada uno de los elementos que definen el sector de intervención.

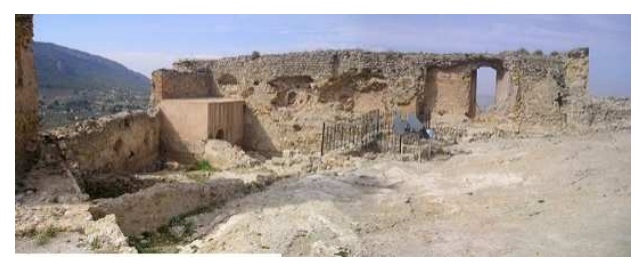

Fig. 2. Espacio interior, previo a la intervención (Miguel del Rey, 2009).

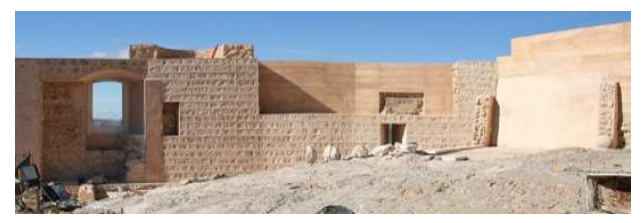

Fig. 3. Espacio interior, tras la intervención (Antonio Gallud, 2010).

\subsection{Recuperación visual de la muralla del re- cinto superior como parte fundamental de la silueta del Castillo}

El indudable interés visual y paisajístico del Castillo de Biar es uno de los aspectos más relevantes de la intervención, por cuanto afianza y define su perfil. La localización del castillo en el punto más elevado le otorga una singularidad visual de carácter prioritario que la restauración de los paramentos de sus murallas, la definición formal de sus torres, así como el recrecido de protección hasta llegar al nivel del adarve, contribuyen a potenciar esa imagen que reside en la memoria del imaginario histórica de la comarca. 


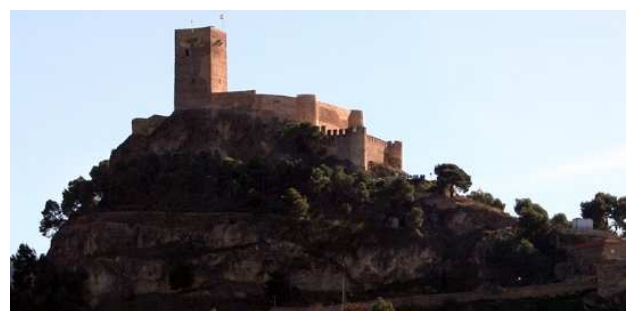

Fig. 4. Recuperación visual del perfil del Castillo (Antonio Gallud, 2010).

\subsection{Mejora de las condiciones de compren- sión de la estructura arquitectónica y de la historia del monumento}

La intervención arquitectónica, más allá de su efecto puramente constructivo, tiene un objetivo de lectura histórica, facilitando la comprensión del monumento y de sus partes, pero haciéndolo de manera que se pueda entender desde escalas y perspectivas distintas. Desde la proximidad del muro o los muros, desde los espacios cerrados que estos definen, o desde las perspectivas externas, tanto próximas como lejanas. Lecturas que pondrán en contexto el perfil de un castillo de frontera, de la belicosa, en cierto momento, frontera entre los Reinos de Castilla y Aragón

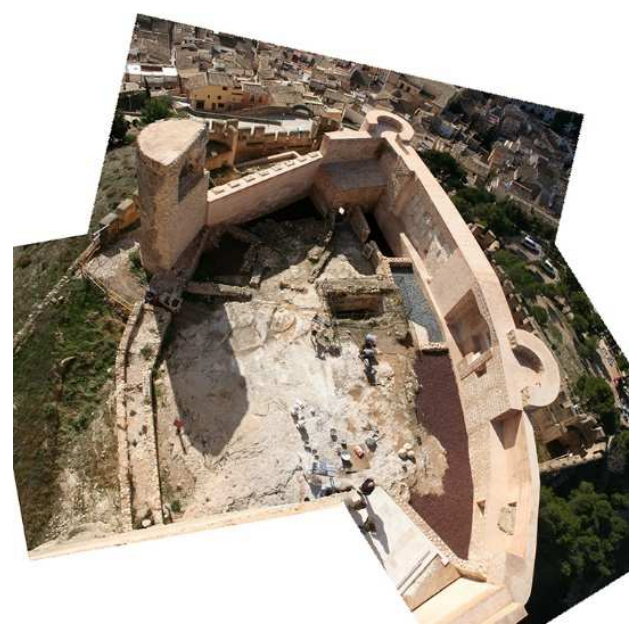

Fig. 5. Visión global de la intervención (Antonio Gallud, 2010).

Dada la riqueza de la información obtenida con las catas realizadas y la presencia física de restos de merlones, adarves, saeteras y parapetos cons- truidos con diversas técnicas según su época; se nos presenta una rica información que permite reconstruir de manera elocuente la cara interna del muro contando en ella toda la historia constructiva del castillo; manteniendo en cambio un perfil más limpio y menos adjetivado en la cara externa del muro, la que se observa desde la visión lejana, la cual permite a su vez reconstruir el perfil coherente del Castillo.

Así pues, quedan reflejadas y con presencia física los restos de este encintado interno del castillo, desde los primeros momentos en época islámica, hasta las sucesivas ampliaciones, mejoras y reconstrucciones que ha tenido el recinto hasta el siglo XVI.

\section{Descripción de las intervenciones en cada fragmento de muro}

\subsection{El muro sur}

Este muro incluye una de las fábricas más heterogéneas que encontramos en esta línea interna de defensa. Se trata de un muro castigado como paramento que incluye en su extremo oeste la puerta de acceso y disponer frente a él del poco espacio posible de ataque, dado lo escarpado del resto de los lados del castillo. La puerta, de época islámica, construida con dovelas formando un arco de medio punto, se encuentra oculta parcialmente por pare de los lienzos de la torre cilíndrica esquinera que fueron ampliando de sección con el tiempo y las diversas reconstrucciones que tuvo el castillo en este primer cinturón hasta el siglo XVI. Las diversas reconstrucciones que, a su vez, tuvo este muro nos muestran en la parte superior un lienzo de fábrica de mampostería heterogénea en forma y tamaño.

Hacia el sureste el lienzo se compone de un basamento de mampostería que nivela el muro sobre la roca madre y sobre el cual se eleva un muro de tapial de mala calidad con relleno de mampuestos no concertados y sin una costra adecuada. Muro de sección escasa ha tenido derribos parciales, en parte por su mala calidad, y también por la escasa sección del mismo. No disponía de adarve de la anchura del muro oeste, más bien se trataba de un pequeño paso que posiblemente en su día fue complementado con una estructura ligera de madera. 


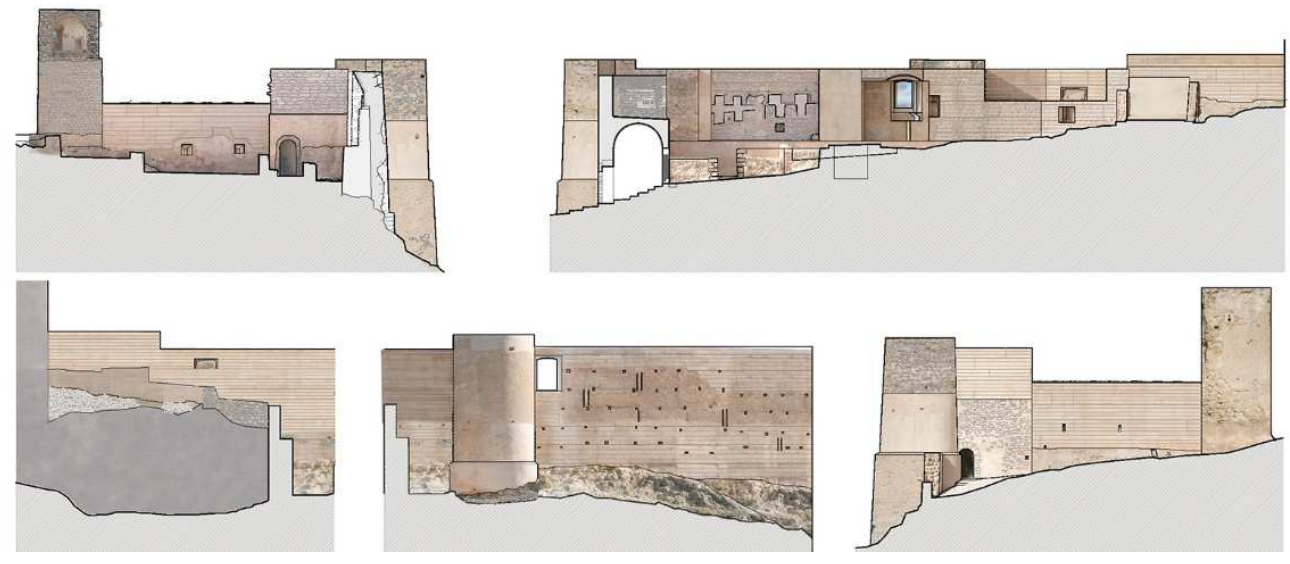

Fig. 6. Alzados interiores y exteriores del Castillo (Miguel del Rey, Antonio Gallud, 2010).

Quedaban huellas de un nivel intermedio que relacionaba este muro sur con el lienzo oeste; nivel hasta al que se ha llegado en la restauración para cerrar esta fachada; mientras que el resto del muro se ha dejado a una altura más baja dado que la fábrica no ofrece, por su esbeltez, todas las garantías de estabilidad mientras no se levante alguno de los muros transversales; muros cuyas bases existen y construían dependencias para estancias de la guardia y que de existir, arriostrarían y el darían estabilidad al flaco sur.

\subsection{La torre sur-suroeste}

La torre cilíndrica que hoy vemos, posiblemente se construyó sobre una antigua esquina en ángulo recto existente en la primera de las cercas islámicas. Torre que se fue ampliando con láminas sucesivas, hasta el extremo de cegar parcialmente la puerta de acceso. Sus acabados son diversos, pues en unos casos se ha terminado con la mampostería directa y en otros su plano cilíndrico estaba revocado con un mortero de cal. En ella se han dejado las improntas de los antiguos muros adosados existentes.

\subsection{El muro oeste}

Especialmente significativo es el caso de este lienzo de muralla donde los enterramientos practicados en su interior a lo largo de los siglos XVIII y XIX, junto a los derrumbes parciales ocurridos por la pérdida de coherencia de los morteros, nos muestran un repertorio de intervenciones de etiología y cronología muy diversa. En él podemos observar la primera de las líneas de defensa del castillo, la musulmana, en su altura original, así como las diversas ampliaciones en altura que se han ido construyendo a lo largo del tiempo, aumentando altura y espesor del lienzo.

Encontramos en el muro oeste tres momentos muy distintos. El primero construido con una mampostería encofrada y tomada con un mortero de cal, sobre la cual se levantan pequeños merlones de mampostería a partir del adarve que se sitúa a escasos metro y medio del nivel interior del patio de armas. Este adarve y parte de los merlones de este nivel se pueden ver en la parte oeste-noroeste. Es la cerca musulmana.

Sobre la línea de este adarve se encuentra una segunda etapa que apoya sus merlones sobre los inferiores, sobreelevado aproximadamente un metro la tapia. Su naturaleza es similar, aunque su acabado era revocado con un mortero de cal. Esta línea se prolonga en otros lienzos de las murallas y de estos niveles quedan restos sobre las fábricas de las torres. Los fragmentos de ambas líneas defensivas se han saneado y dejado vistos en la cara interna del muro oeste. Línea que posiblemente corresponde a un momento de transición entre la dominación musulmana a la cristiana. 


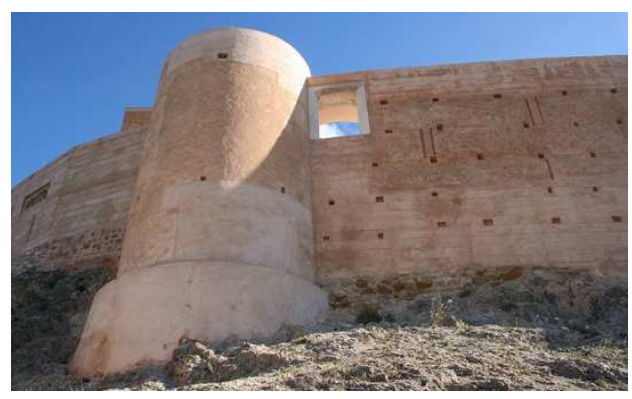

Fig. 7. Muro oeste y torre (Antonio Gallud, 2010).

La última ampliación en altura y espesor de las murallas se realiza ya en época cristiana, cuando se realizan diversas intervenciones con fábricas y acabados muy diversos: tapiales en el encofrado de la lámina externa que sube hasta el adarve más alto. Una lámina de mampostería engrosa la antigua sección en la cara interna, ocultando las fábricas más antiguas. Sobre este muro, y en su última etapa en activo, en época medieval, se abren diversas estancias hacia el patio de armas, a la vez que una ventana palaciega, posiblemente la casa del Alcayde, abre a poniente; ventana de gran dimensión y con un parteluz central. Ventana de la que quedan el alfeizar y las jambas y el arco superior de descarga; elementos que se han recuperado y restaurado, así como el "festejador" lateral derecho.

Este lienzo oeste ha recuperado la altura del adarve, sin levantar el posible parapeto, ya que de él no se tiene noticia ni restos en esta parte del lienzo para poder reproducirlo. Las torres se han dejado ligeramente más altas que el nivel del paso de guardia, tal como mostraban los restos existentes.

La sección de muro por la parte externa presenta el ligero talud original, mientras que por el interior se han practicado vaciados que permitan dejar vistos los distintos momentos de construcción de la muralla, que hemos comentado anteriormente. Lo cual ha obligado a recuperar en la parte alta el espesor de la última etapa del muro y mostrar el ancho del adarve de esta época, dejando una sección variable de una cierta elocuencia que en ocasiones presenta un plano inclinado en disminución hacia la parte media de la altura del muro.
Las técnicas constructivas se han respetado en cada uno de los puntos, resolviéndose el lienzo exterior con un encofrado de madera de tabla lisas y ancho de palmo valenciano, conservando amplias zonas de muro original con su textura y acabado, preparando las bases de los paramentos mediante aplicación de base de silicato diluido, como se indicó en el caso del muro sur. De la misma manera se han tratado las fisuras y concavidades en los paramentos conservados en su acabado original. Por último, se han tonificado el paramento y se impermeabilizó, tal como se descrito anteriormente.

\subsection{El muro norte}

El muro en el tramo primeramente descrito, por su parte interna, se ha levantado hasta el nivel del adarve islámico original con el material como este fue construido, con mampostería encofrada; mientras que a partir de esta altura se levanta con un tapial de mortero de cal bastardo. Por la parte externa el muro presenta toda su altura y se ha restaurado con el encofrado de tabla de palmo valenciano y fábrica de tapial de mortero de cal bastardo. En la parte baja de este muro encontramos una saetera de amplia dimensión, posiblemente de época cristiana, que defendía la parte externa del lienzo del primer recinto amurallado

En este punto y por la parte externa se han encontrado restos de lo que pudiera haber sido una torre esquinera levantada sobre un saledizo de la roca y que se mantiene visible tras la intervención.

El resto del muro, hasta llegar a la torre celoquia, se ha restaurado a la manera del tapial, tal como mostraban sus fábricas, rehaciendo el adarve existente, con toda su anchura y repuesto el parapeto, del cual quedaban restos ciertos. Se han dejado sobre los muros interiores las improntas de los muros de lo que fue la ermita o la capilla posterior del cementerio.

\subsection{La torre oeste}

Esta torre muestra una sección uniforme en toda su altura, es de forma semicilíndrica en el exterior y plana en la parte interna, permitiendo el acceso de los adarves de los muros que llegan 
hasta ella. En su interior se encuentran restos de la antigua cerca original, que pasa por detrás de la torre, lo cual nos indica que en origen no existía torre. En ella hubo hace unos años una intervención de una Escuela Taller. No ha habido intervención en la misma por no entrar en presupuesto.

\subsection{El muro noroeste}

Este muro tiene una traza curvilínea con una pequeña inflexión cuando cambia de noroeste a norte. Presentaba un estado de ruina total en la parte adosada a la torre noroeste y hasta el punto donde se encontramos la impronta de la antigua ermita. Desde aquí, el muro mantenía una sección algo más coherente, al menos en altura, donde era posible entrever por donde pasaba el adarve y como eran sus protecciones, que en este caso era un parapeto. Las fábricas eran de tapial, similares a las de la parte este del muro sur. No ha habido intervención sobre este lienzo por no entrar en presupuesto.

\section{Técnicas utilizadas}

Cada una de las fábricas se ha restaurado siguiendo su propia naturaleza y utilizando una combinación de técnicas tradicionales y productos de alta calidad en intervenciones concretas: consolidación de bases de paramentos, sellado y relleno de fisuras con morteros especiales, anclajes de las diversas láminas de muros, tonificación e impermeabilización de los muros, etc.

Las técnicas constructivas y la métrica de encofrados se han respetado en cada uno de los puntos, resolviéndose los cofres de tapiales con listón de madera de tabla lisas y ancho de palmo valenciano, conservando los morteros, acabados y texturas donde este existía, preparando las bases de los paramentos cuando debían incorporarse faltantes realizado mediante aplicación de base de silicato diluido en una pequeña parte de agua para mejorar la penetración del material, mientras que para el tratamiento de fisuras se han realizado inyecciones que incluían una base de lechada de cal hidráulica/cal apagada amasada con resina acrílica diluida en agua que, según el espesor de la fisura, han variado las proporciones de cal hidráulica con cal apagada. Para el tratamiento de micro fisuras, como para la consolidación de paramentos con grafitis, se emplearon bases de silicato y polvo de mármol para poder dar la dureza necesaria, con la fluidez oportuna que permita una buena penetración del material.

En las zonas erosionadas se ha repuesto el plano con un encofrado y mortero bastardo de cal coloreado con la propia arena, de tono muy similar al original. Cromatismo que más tarde se ha tonificado con diluyentes de silicato. Diluido en agua utilizando los colorantes específicos del silicato, se elabora una base del color a tratar aplicando dos o tres manos hasta conseguir la igualación y entonación del mortero a tratar. Cuando la base es más oscura que la entonación de la superficie se utiliza base de silicato en pasta y se diluye hasta conseguir la entonación adecuada.

Por último, se han impermeabilizado los muros, en especial los planos superiores y salientes. La impermeabilización en coronación se realizó mediante aplicación de hidrofugante por pulverización 3 manos hasta saturación. El material empleado se elaboró en las instalaciones de la empresa, a base de un hidrofugante a base de siloxanos diluido en disolvente sin olor.

Respecto a los grafitis encontrados, el conjunto más interesante corresponde a dos barcos grabados en uno de los pocos lienzos que no fueron enfoscados con cemento en la restauración de los años setenta, concretamente en la muralla que queda a la izquierda al acceder al recinto superior. Asociados a estos barcos, también hay incisos una bandera, una ballesta y una estrella de cinco puntas. Incisión situada en el recinto superior IV, Cronología Bajomedieval (finales de siglo XII - siglo XIV) Conjunto de dimensiones: $1,8 \times 0,8 \mathrm{~m}$. (aproximadamente). Todo ello se ha restaurado y estabilizado.

\section{Conclusiones}

Durante el periodo inicial la actitud reflexiva, pausada y atenta nos llevaba a analizar los lienzos de muralla buscando indicios o huellas que nos ayudaran a conocer la historia vivida por esos muros. 
El castillo es el resultado de la superposición de sistemas y elementos constructivos en aparente desorden y, en muchos casos, ejecutados a toda prisa, quizá en el intermedio entre un ataque y el próximo inminente. La labor conjunta de miradas atentas por parte del equipo redactor, los datos históricos y la labor del arqueólogo consigue poner relativamente en orden la historia de los muros. En ese momento es necesario dar coherencia formal a esa historia para que sea capaz de ser transmitida.

Finalmente, el resultado de la intervención se convierte en un lienzo donde quedan claramente reflejados el paso del tiempo y sus técnicas constructivas.

\section{Bibliography}

Azuar, R. (1981). Castellología medieval alicantina. Área meridional, Instituto de Estudios Alicantinos Ed., Alicante.

Azuar, R. (1983). "Panorama de la arqueología medieval de los valles alto y medio del Vinalopó (Alicante)", in Lucentum. II, Universidad de Alicante Ed., Alicante, pp. 349-383.

Bérchez, J., et al. (1983). Catálogo de monumentos y conjuntos de la Comunidad Valenciana, Conselleria de Cultura, Educación y Ciencia, Generalitat Valenciana Ed., Valencia.

Brandi, C. (1999). Teoría de la restauración, Alianza Ed., Madrid.

Capitel, A. (1988). Metamorfosis de monumentos y teorías de la restauración, Alianza Ed., Madrid.

Font, F.; Hidalgo, P. (1990). El tapial: una técnica constructiva milenaria, Colegio Oficial de Aparejadores y Arquitectos Técnicos de Castellón Ed., Castellón.

Guichard, P. (1982). "Los castillos musulmanes del norte de la provincia de Alicante", in Anales de la Universidad de Alicante. Historia medieval, Universidad de Alicante Ed.

Guinot, E. (1995). Els límits del Regne: el procés de formació territorial del País Valencià medieval (1238-1500), Alfons El Magnànim Ed., València.

Hinojosa, J. (1995). Biar, un castillo de la frontera valenciana en la Edad Media, Diputación Provincial de Alicante Ed., Alicante.

Jiménez, J. (1995). El castillo medieval español y su evolución, Agualarga Ed., Madrid.

Rey, M. Del.; Gallud, A. (2011). Intervención y consolidación del segundo recinto amurallado del Castillo de Biar, UPV Ed., Valencia.

Segura, G.; Simón, J.L. (2001). "Castillo de Biar", in Castillos y torres en el Vinalopó. Col-leecció l'Algoleja, 4, Centre d'Estudis Locals del Vinalopó Ed., Petrer, pp. 55-60.

Tendero, F. (2003). "Castillo de Biar", in Actuaciones arqueológicas en la provincia de Alicante, Sección de Arqueología del Ilustre Colegio Oficial de Doctores y Licenciados en Filosofía y Letras y en Ciencias de Alicante Ed.

Tendero, F. (2009). "Castillo de Biar", in Graffiti arte espontáneo en Alicante, Museo Arqueológico de Alicante MARQ Ed., Alicante.

Torró, J. (2012). "La conquista del reino de Valencia. Un proceso de colonización medieval desde la arqueología del territorio", in La conquista de Al-andalus en el siglo XIII, Centro de Estudios Medievales Ed., Murcia. 